\title{
Docking Haptics: Dynamic Combinations of Grounded and Worn Devices
}

\author{
Anthony Steed; Sebastian Friston, Vijay Pawar, David Swapp \\ Department of Computer Science, University College London, United Kingdom
}
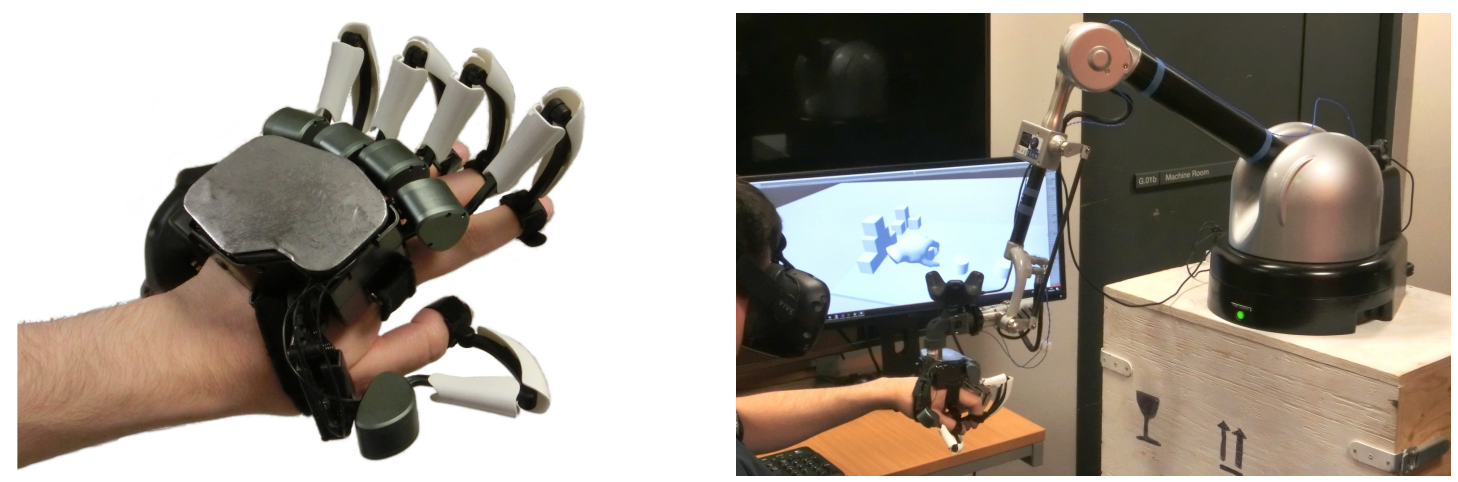

Figure 1: In our prototype implementation of docking haptics, the user wears a hand exoskeleton (Dexmo) with an attached mild steel plate (Left). Right: As they approach an area where the simulation desires to create the impression of weight, the exoskeleton is dynamically attached to a grounded six degree-of-freedom robot, a Haption Virtuose 6D, that applies forces through the plate to the hand exoskeleton.

\begin{abstract}
Grounded haptic devices can provide a variety of forces but have limited working volumes. Wearable haptic devices operate over a large volume but are relatively restricted in the types of stimuli they can generate. We propose the concept of docking haptics, in which different types of haptic devices are dynamically docked at run time. This creates a hybrid system, where the potential feedback depends on the user's location. We show a prototype docking haptic workspace, combining a grounded six degree-of-freedom force feedback arm with a hand exoskeleton. We are able to create the sensation of weight on the hand when it is within reach of the grounded device, but away from the grounded device, hand-referenced force feedback is still available.
\end{abstract}

Index Terms: Human-centered computing-Haptic Devices-; Human-centered computing-Virtual Reality-;

\section{INTRODUCTION}

There are many types of haptic force-feedback controller. The Rutgers Master II was used in Virtual Reality (VR) in the mid 1990s [2] It is still characteristic of hand-mounted devices where a robot with multiple articulations is attached to the outside or inside of the user's hand. The main limitation of such devices is that they can't provide external forces, e.g. sensation of weight of an object. In contrast, a grounded device such as the Phantom [5] is typically mounted attached to a fixed base enabling the device to transmit a net force to the user.

We propose the concept of docking haptics - a class of hybrid

*a.steed@ucl.ac.uk system that enables the dynamic re-configuration of different types of haptic devices to extend their workspace and capabilities, see Figure 1. We suggest that by combining wearables or hand-helds with grounded devices we can extend the system capabilities beyond those of the individual devices and provide plausible feedback in a broader range of immersive simulations.

\section{Docking Haptics Concept}

The docking haptics concept proposes that by dynamically docking haptic devices we can explore a much larger design space for haptic feedback. By docking, we mean one device attaching to another with a temporary joint. The joint may be rigid, or have some Degrees of Freedom (DOFs).

While some types of multi-part shape-changing robot fall under this definition (e.g. [6]) we are interested in exploring the space by combining the best capabilities of different classes of device.

Docking creates a connection between two bodies. If we consider this as a mechanical problem, we would be forming kinematic pairs [4]. Examples of such pairs include revolute or hinged joints, prismatic joints and planar joints. However, we want to effect the joint temporarily, so we could consider mechanisms such as electromagnetic, hydraulic or mechanical linkages.

\section{Prototype Implementation}

We constructed a proof-of-concept dockable haptic workspace. The wearable was a Dexmo glove and the arm was a Haption Virtuose 6D with an electromagnet-based docking mechanism. Both units were controlled by the same Unity application. The complete apparatus is shown in Figure 1:Right.

\subsection{Hardware}

The Dexmo glove by Dexta Robotics (Figure 1:Left, with our modifications) is a new iteration of a passive-admittance based device 
presented by $\mathrm{Gu}$ et al. [3]. The current version has 5 force-feedback DOFs, 11 sensed DOFs, and 10 uninstrumented DOFs.

The Virtuose 6D by Haption is a robotic arm designed for groundfixed tool based haptics. It has $6 \mathrm{DOF}$, able to set both tool position and orientation within its workspace. The Virtuose supports both impedance and admittance-based control. We used the latter.

The Virtuose is dynamically attached to the hand using an electromagnet. The Dexmo cover was replaced with a mild-steel plate. The usual Virtuose tool was replaced with an attachment hosting the magnet and a tracker for controlling open-loop docking. The magnet power supply was controlled with simple serial commands to a microcontroller over USB. Both the Dexmo and the end-effector of the Virtuose controller were tracked using Vive pucks.

\subsection{Software}

The Virtual Environment (VE) was constructed in Unity 2018 and used the inbuilt PhysX engine to perform the simulation. The Dexmo glove provides a managed library that was integrated directly with Unity. The Virtuose C library was thinly wrapped with P/Invoke. The Dexmo should not be updated at more than $30 \mathrm{~Hz}$ while the Virtuose must be updated at no less than $30 \mathrm{~Hz}$. The Dexmo and magnet controller ran in the main Unity thread, while the Virtuose SDK ran its own thread to issue callbacks, and in these callbacks we implemented force control and tracking \& interception, based on parameters set from the main thread. The hand was modelled by a set of colliders that determined forces relayed to both Dexmo and Virtuose.

The key part of the software is the tracking and interception of the plate on the Dexmo by the magnet on the Virtuose. For this prototype we simply attempted to match the transform of the magnet to a target point on the hand (Pure Pursuit). This interception task is the main avenue for future work in the short term.

\subsection{Demonstration Environment}

We combined the docking haptics system with a VR headset, the HTC Vive. Thus, the user can move freely around a large workspace wearing the Dexmo, but when close to the Virtuose (seated in our demonstration, see Figure 1), the two haptic devices dock.

We implemented a simple demonstration where users could manipulate three identical cans with different weights, see Figure 2. In a pilot trial users were able to distinguish between weights when the device was docked, but were not when using the Dexmo only.

\section{Discussion and Future Work}

Our design uses a simple connector between the two devices. We can imagine that various different connectors could be made such as linear tracks, rigid connections (the current magnetic connection leaves one axis of rotation) and rotational joints.

Where rigid docking is used, robot range-of-motion will become perhaps the most important characteristic. Active compensation is necessary for any practical system, as the Dexmo glove and robot together are heavy. Active compensation may require more precise control, but it could also be performed with force sensors only, which the Virtuose supports.

Future implementations will need better integration in order to

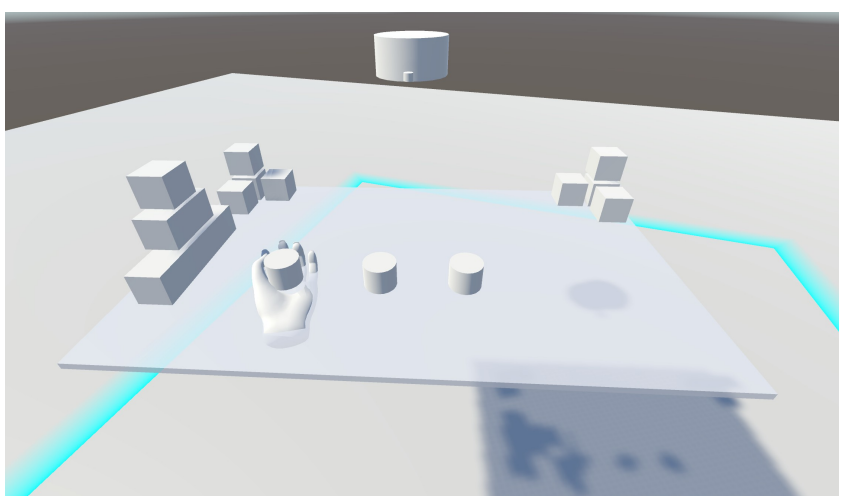

Figure 2: Simple example environment

render physically correct forces. Game-optimised engines may have difficulty with complex arrangements of colliders like those of the hand, resulting in distracting jitter and extreme responses.

Another area to explore is the combination of docking with haptic retargeting [1]. Given that joints may be stronger in some directions than others, and that users seem relatively insensitive to small relative position offsets, we can perhaps construct docking configurations that appear to generate forces from a wider range of directions and strengths than is physically realizable.

\section{Conclusion}

In docking haptics, a system dynamically couples different components at run-time to build the most appropriate combination for a particular task and environment.

We built a demonstrator that proved the concept viable and highlighted some challenges to develop this concept. There is a very large design space to explore, including active and passive haptics, as well as other types of grounded and encumbering devices.

\section{REFERENCES}

[1] M. Azmandian, M. Hancock, H. Benko, E. Ofek, and A. D. Wilson. Haptic Retargeting: Dynamic Repurposing of Passive Haptics for Enhanced Virtual Reality Experiences. In Proceedings of the 2016 CHI Conference on Human Factors in Computing Systems, CHI '16, pp. 1968-1979. ACM, 2016.

[2] D. Gomez, G. Burdea, and N. Langrana. Integration of the Rutgers Master II in a virtual reality simulation. In Proceedings Virtual Reality Annual International Symposium '95, pp. 198-202, 1995.

[3] X. Gu, Y. Zhang, W. Sun, Y. Bian, D. Zhou, and P. O. Kristensson. Dexmo: An Inexpensive and Lightweight Mechanical Exoskeleton for Motion Capture and Force Feedback in VR. Proceedings of the 2016 CHI Conference on Human Factors in Computing Systems - CHI '16, pp. 1991-1995, 2016. doi: 10.1145/2858036.2858487

[4] R. S. Hartenberg and J. Denavit. Kinematic synthesis of linkages. McGraw-Hill, New York, 1964

[5] T. H. Massie and J. K. Salisbury. The phantom haptic interface: A device for probing virtual objects. In Proceedings of the ASME winter annual meeting, symposium on haptic interfaces for virtual environment and teleoperator systems, vol. 55, pp. 295-300. Citeseer, 1994.

[6] Y. Zhao, L. H. Kim, Y. Wang, M. Le Goc, and S. Follmer. Robotic Assembly of Haptic Proxy Objects for Tangible Interaction and Virtual Reality. In Proceedings of the 2017 ACM International Conference on Interactive Surfaces and Spaces, ISS '17, pp. 82-91. ACM, 2017. 